\title{
Combinational Effect of Cell Adhesion Biomolecules and Their Immobilized Polymer Property to Enhance Cell-Selective Adhesion
}

\author{
Rio Kurimoto, ${ }^{1,2}$ Kei Kanie, ${ }^{3}$ Naokazu Idota, ${ }^{4}$ Mitsuo Hara, ${ }^{5}$ \\ Shusaku Nagano, ${ }^{6}$ Takehiko Tsukahara, ${ }^{7}$ Yuji Narita, ${ }^{8}$ Hiroyuki Honda, ${ }^{9}$ \\ Masanobu Naito, ${ }^{1,10}$ Mitsuhiro Ebara, ${ }^{2}$ and Ryuji Kato ${ }^{3}$ \\ ${ }^{1}$ Graduate School of Pure and Applied Sciences, University of Tsukuba, 1-1-1 Tennodai, Tsukuba, Ibaraki 305-8577, Japan \\ ${ }^{2}$ Biomaterials Unit, International Center for Materials Nanoarchitectonics (WPI-MANA), National Institute for \\ Materials Science (NIMS), 1-1 Namiki, Tsukuba, Ibaraki 305-0044, Japan \\ ${ }^{3}$ Department of Basic Medicinal Sciences, Graduate School of Pharmaceutical Sciences, Nagoya University, Furo-cho, \\ Chikusa-ku, Nagoya, Aichi 464-8601, Japan \\ ${ }^{4}$ Kagami Memorial Research Institute for Materials Science and Technology, Waseda University, 2-8-26 Nishiwaseda, \\ Shinjuku-ku, Tokyo 169-0051, Japan \\ ${ }^{5}$ Department of Molecular Design and Engineering, Graduate School of Engineering, Nagoya University, Furo-cho, \\ Chikusa-ku, Nagoya, Aichi 464-8603, Japan \\ ${ }^{6}$ Nagoya University Venture Business Laboratory, Nagoya University, Furo-cho, Chikusa-ku, Nagoya, Aichi 464-8603, Japan \\ ${ }^{7}$ Research Laboratory for Nuclear Reactors, Tokyo Institute of Technology, 2-12-1 Ookayama, Meguro-ku, \\ Tokyo 152-8550, Japan \\ ${ }^{8}$ Department of Cardiac Surgery, Nagoya University Graduate School of Medicine, 65 Tsurumai-cho, Showa-ku, Nagoya, \\ Aichi 466-8550, Japan \\ ${ }^{9}$ Department of Biotechnology, Graduate School of Engineering, Nagoya University, Furo-cho, Chikusa-ku, Nagoya, \\ Aichi 464-8603, Japan \\ ${ }^{10}$ Structural Materials Unit, Research Center for Strategic Materials, National Institute for Materials Science (NIMS), \\ 1-1 Namiki, Tsukuba, Ibaraki 305-0044, Japan
}

Correspondence should be addressed to Ryuji Kato; kato-r@ps.nagoya-u.ac.jp

Received 17 February 2016; Revised 2 May 2016; Accepted 5 May 2016

Academic Editor: Matthew Green

Copyright (c) 2016 Rio Kurimoto et al. This is an open access article distributed under the Creative Commons Attribution License, which permits unrestricted use, distribution, and reproduction in any medium, provided the original work is properly cited.

Although surface immobilization of medical devices with bioactive molecules is one of the most widely used strategies to improve biocompatibility, the physicochemical properties of the biomaterials significantly impact the activity of the immobilized molecules. Herein we investigate the combinational effects of cell-selective biomolecules and the hydrophobicity/hydrophilicity of the polymeric substrate on selective adhesion of endothelial cells (ECs), fibroblasts (FBs), and smooth muscle cells (SMCs). To control the polymeric substrate, biomolecules are immobilized on thermoresponsive poly $(N$-isopropylacrylamide-co-2carboxyisopropylacrylamide) (poly(NIPAAm-co-CIPAAm))-grafted glass surfaces. By switching the molecular conformation of the biomolecule-immobilized polymers, the cell-selective adhesion performances are evaluated. In case of RGDS (Arg-Gly-AspSer) peptide-immobilized surfaces, all cell types adhere well regardless of the surface hydrophobicity. On the other hand, a tri-Argimmobilized surface exhibits FB-selectivity when the surface is hydrophilic. Additionally, a tri-Ile-immobilized surface exhibits EC-selective cell adhesion when the surface is hydrophobic. We believe that the proposed concept, which is used to investigate the biomolecule-immobilized surface combination, is important to produce new biomaterials, which are highly demanded for medical implants and tissue engineering. 


\section{Introduction}

For long-term implantation of medical devices, such as vascular grafts, both cellular-compatibility and antithrombogenicity are essential $[1,2]$. The realization of functional sustainability has been a challenge for biomaterial chemistry to minimize life-threatening risks.

One major strategy to provide biological functionality (e.g., cell adhesion) for medical applications of polymer materials is immobilization of biomolecules using extracellular matrix (ECM) proteins [3-6], antibodies [7], peptides [8-13], and so forth. Cell adhesion is a critical step to regenerate tissues damaged by implantation surgery. For example, endothelialization of vascular grafts can be enhanced by improving adhesion of endothelial cells (ECs) on their surface. Faster endothelialization prevents two risks of restenosis: thrombosis and neointimal hyperplasia [14-16]. The monolayer coverage of endothelial cells strongly provides antithrombosis effect. The appropriate endothelial monolayer formation is also the first step of proper cellular organization, which inhibits the uncontrolled growth of smooth muscle cells and fibroblasts that invade from the outer layer of vascular tissue.

Peptides on polymers have shown successful cell adhesive function. RGD (Arg-Gly-Asp), which is the integrin ligand sequence, is one of the most well established biomolecules that provide a cell adhesion function on polymers [17]. Moreover, various peptides from ECMs exhibit a cell adhesion performance. We have reported that short peptides $(3 \mathrm{mer}$ to $7 \mathrm{mer}$ ) enhance not only cell adhesion [18] but also cellselective adhesion $[19,20]$. An ECM-derived 3 mer peptide (CAG: Cys-Ala-Gly) enhances EC adhesion for endothelialization but inhibits smooth muscle cell (SMC) adhesion inside the electrospun vascular scaffold composed of poly- $\varepsilon$ caprolactone in vivo [21] and inhibits platelet adhesion on a material surface [22].

Through our systematic cell adhesion peptide screening, we have found two classes of adhesion peptides. One exhibits a strict ligand-receptor behavior (RGDS and YIGSR). The other is more closely related to motifs that largely depend on their physiochemical properties and therefore may accept a range of sequence variations. That is, not only the sequence itself [cell-peptide interaction through ligand-receptor (e.g., RGD peptide)] but also the physicochemical property can control cell-selective adhesion [19].

Ile-containing peptides ( 3 mer to 7 mer) show selective adhesion of ECs, but they show a negative adhesion effect on SMCs and fibroblasts (FBs). Compared to antibodies and ECM-derived large proteins, short peptides can be feasibly synthesized and purified. The assurance of their purity and production costs is advantageous for functionalizing medical materials, but similar to other biological molecules, peptides require skillful immobilization to sustain their original performance. There are several unknown factors that can diminish biomolecule performance, including side effects of immobilization chemistry, structural/directional changes by immobilization, molecular density of immobilization, or combinational effects of biomolecule-polymer compatibility. Understanding the cause of such unexpected effects in biomolecule immobilization is important when designing medical devices.

Additionally, the properties of the polymer itself greatly influence cell adhesion. Wettability [23], elasticity [24], and geometry [25] of the polymer material are critical factors affecting biocompatibility. Previously, we reported that the level of hydrophobicity of polyNIPAAm can change the cell adhesion performance of the same immobilized-RGDS (ArgGly-Asp-Ser) peptide [26-28].

Based on these observations, we hypothesize that there should be a combinational effect of biomolecules and the immobilized polymer status that may inhibit/enhance the total functionality. To investigate such a biomolecule-polymer combinational effect, we designed a cell assay platform that can compare the total performance of the target biomolecules combined with different polymer properties by applying thermoswitchable polyNIPAAm technology (Figure 1). Altering the status of the biomoleculeimmobilized polyNIPAAm by temperature allows the performance of the same immobilized biomolecule to be assayed as a function of the polymer properties.

In this work, we apply the cell assay platform of poly(NIPAAm-co-CIPAAm) [26-29] to compare cell adhesion performances. Specifically, the changes in the cell adhesion performance and the preference among three different cell types (ECs, FBs, and SMCs), which we refer to as "cell-selective adhesion" (Figure 1), are evaluated. The results indicate that immobilized biomolecule performances depend on the combination of the polymer status and appropriately selecting the immobilization polymer may sustain or even enhance the cell-selective adhesion performance of biomolecules.

\section{Materials and Methods}

2.1. Preparation of the Poly(NIPAAm-co-CIPAAm) Cell Assay Platform. The poly(NIPAAm-co-CIPAAm) cell assay platform was prepared using a surface-initiated atom transfer radical polymerization (ATRP) technique [29] (Figure 2). Water-repellent printed slide glasses and regular slide glasses $(10 \mathrm{~mm} \times 25 \mathrm{~mm})$ (Matsunami Glass Industry, Tokyo, Japan) were exposed to UV ozone for $10 \mathrm{~min}$ for cleaning. Then the clean glass platform was placed in a glass container with (chloromethyl)phenylethyl-trimethoxysilane (Gelest, Inc., Morrisville, PA, USA) for the silanization reaction for $3 \mathrm{~h}$ at $90^{\circ} \mathrm{C}$ and subsequently baked for $1 \mathrm{~h}$ at $110^{\circ} \mathrm{C}$. NIPAAm (Kohjin, Tokyo, Japan) was purified by recrystallization from $n$-hexane (Wako Pure Chemical Industries, Osaka, Japan).

2-(Benzyloxycarbonyl)isopropylacrylamide (CIPAAmBz) was synthesized and purified according to the previously reported protocol [30]. On the surface of the silanized glass platform, a poly(NIPAAm-co-CIPAAmBz) layer was grafted in 2-propanol (Kanto Chemical, Tokyo, Japan) with a molar ratio of 100:1 (NIPAAm:CIPAAmBz) with a free ATPR initiator at room temperature for $17 \mathrm{~h}$. In a typical procedure, $17.924 \mathrm{~g}(2 \mathrm{~mol} / \mathrm{L})$ of NIPAAm, $401 \mathrm{mg}$ $(20 \mathrm{mmol} / \mathrm{L})$ of CIPAAmBz, $72 \mathrm{mg}(8.99 \mathrm{mmol} / \mathrm{L})$ of $\mathrm{Cu}(\mathrm{I}) \mathrm{Cl}$ (Kanto Chemical), and $10 \mathrm{mg}(1.00 \mathrm{mmol} / \mathrm{L})$ of $\mathrm{Cu}(\mathrm{II}) \mathrm{Cl}_{2}$ (Wako Pure Chemical Industries) were dissolved in $80 \mathrm{~mL}$ of 


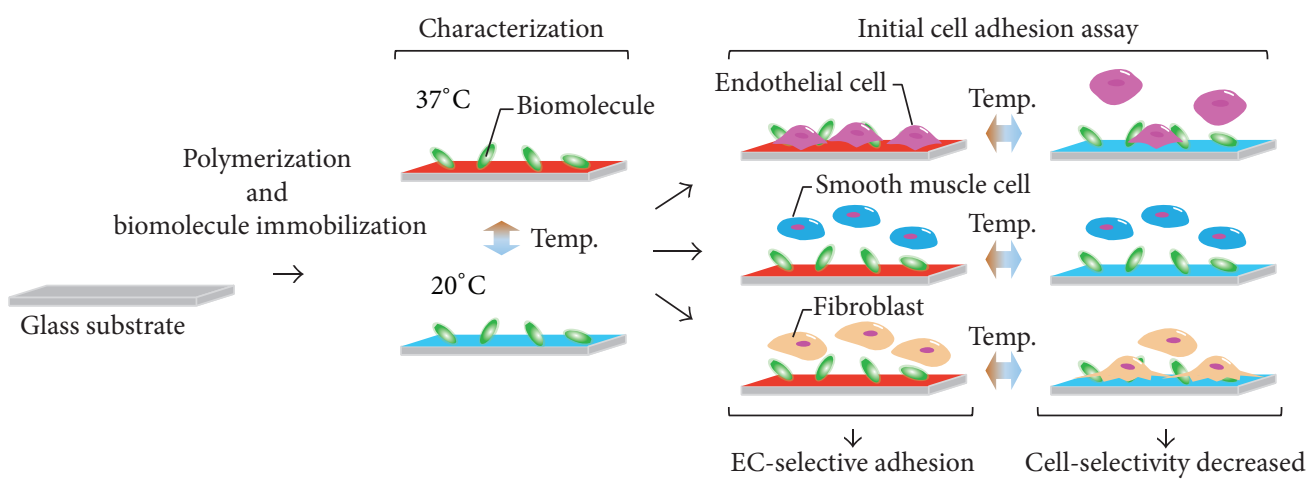

FIGURE 1: Schematic image of evaluating cell adhesion performance as a combination effect of biomolecules and its immobilized polymer property. Poly(NIPAAm-co-CIPAAm) surface was fabricated to create the cell assay platform. Due to the thermoresponsive polyNIPAAm effect, the cell adhesion performance of the same immobilized biomolecules can be tested; the polymer properties can be compared by changing the temperature. Cell-selective adhesion was compared among three types of cells (ECs, SMCs, and FBs), which control the regeneration process of vascular tissue engineering.
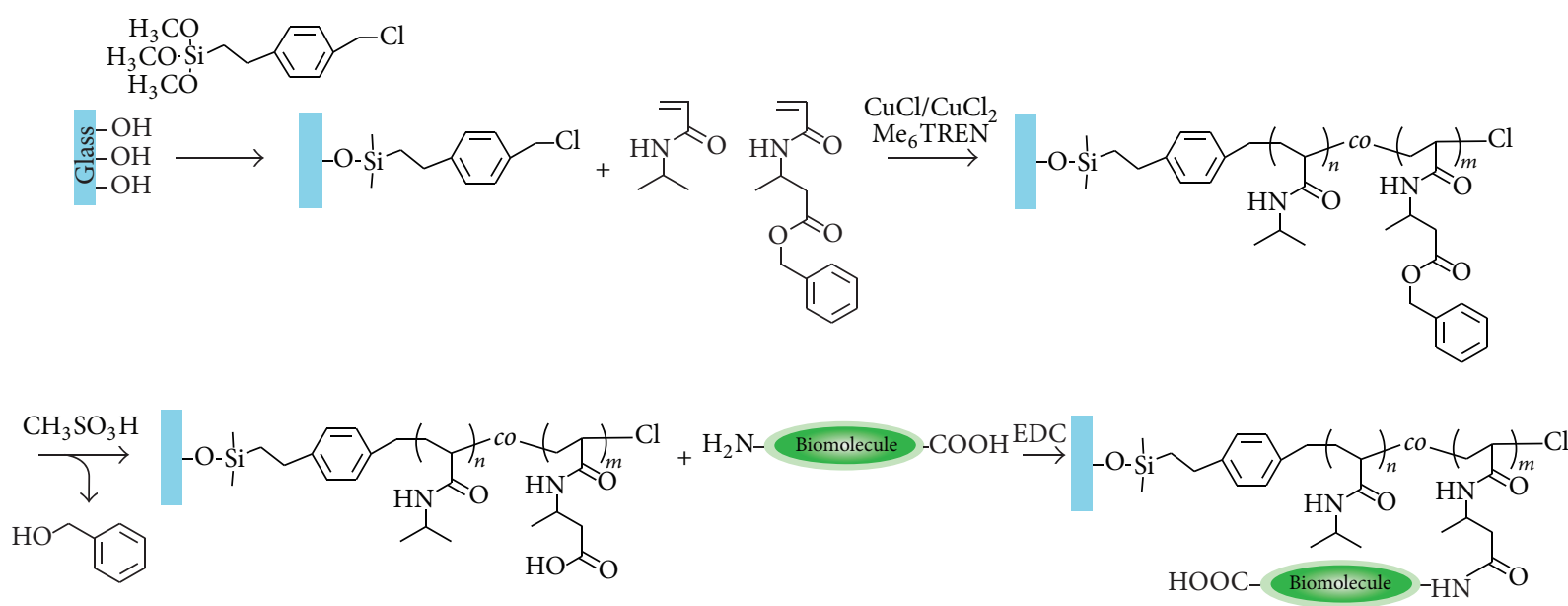

FIGURE 2: Fabrication scheme for the poly(NIPAAm-co-CIPAAm) graft and biomolecule immobilization.

the degassed solvent in a glove box under a nitrogen atmosphere. The silanized glasses, $200 \mathrm{mg}(10.8 \mathrm{mmol} / \mathrm{L})$ of $\mathrm{Me}_{6}$ TREN (Mitsubishi Chemical Holdings, Tokyo, Japan) and $133 \mathrm{mg}(10.7 \mathrm{~mol} / \mathrm{L})$ of 4-ethylbenzyl chloride (Tokyo Chemical Industry, Tokyo, Japan), were added to the above solution. The reaction was terminated by exposing the solution to air. The polymer layered platform was rinsed with 2-propanol and methanol (Kishida Chemical, Osaka, Japan) and dried under a vacuum. Deprotection of CIPAAmBz in the polymer layer was carried out by acidic hydrolysis with methanesulfonic acid (Wako Pure Chemical Industries). In a typical procedure, $37.5 \mathrm{~mL}$ ( $15 \mathrm{vol} \%$ ) of methanesulfonic acid was dissolved in $212.5 \mathrm{~mL}$ of dichloromethane (Kanto Chemical). The polymer-grafted cell assay platform was deprotected by acidic hydrolysis with 15 vol\% methanesulfonic acid in dichloromethane at room temperature for $17 \mathrm{~h}$. The activated platform surface was then rinsed with dichloromethane and methanol for the biomolecule immobilization step.

To check the quality of the synthesized polymer, the molecular weight of the free polymer was determined by a gel permeation chromatography (GPC) system (TOSOH TSKGEL $\alpha-2500$, Tosoh, Tokyo, Japan) equipped with a refractive index detector (RI-2031) at $40^{\circ} \mathrm{C}$. The polymer solution was first dialyzed with pure water and then applied to GPC. The GPC measurement confirmed that the molecular weight $\left(M_{n}\right)$ was $6.95 \times 10^{3} \mathrm{~g} / \mathrm{mol}$ (Table 1$)$.

2.2. Biomolecule Immobilization on Poly(NIPAAm-coCIPAAm) Cell Assay Platform. Biomolecules (di- or tetrapeptides) were immobilized onto CIPAAm via the carboxyl groups with an equal molar amount of 1-ethyl-3-(3dimethylaminopropyl)carbodiimide hydrochloride (EDC) (Dojindo Laboratories, Kumamoto, Japan) in pure water under a humid atmosphere at room temperature for $17 \mathrm{~h}$. Then the platform was rinsed with pure water and dried under a vacuum until cell assay usage. Gly-Arg (mono-Arg), Gly-Ile (mono-Ile), Gly-Arg-Arg-Arg (tri-Arg), and GlyIle-Ile-Ile (tri-Ile) (>90\% purity) synthesized by Biomatik (Cambridge, ON, Canada) and RGDS ( $>98 \%$ purity) purchased from Peptide Institute (Osaka, Japan) were used as biomolecules for immobilization. 
TABLE 1: Characterization of the grafted polymer.

\begin{tabular}{|c|c|c|c|c|}
\hline Thickness $(\mathrm{nm})^{\mathrm{a}}$ & Density $\left(\mathrm{g} / \mathrm{cm}^{3}\right)^{\mathrm{a}}$ & $M_{n} \times 10^{-3 b}$ & $M_{w} / M_{n}{ }^{\mathrm{b}}$ & Graft density (chains/nm $\left.{ }^{2}\right)^{\mathrm{c}}$ \\
\hline 3.66 & 0.95 & 6.95 & 1.21 & 0.30 \\
\hline
\end{tabular}

2.3. Characterization of the Biomolecule-Immobilized Poly(NIPAAm-co-CIPAAm) Cell Assay Platform. To characterize the cell assay platform, X-ray reflectivity (XRR) was performed with a Rigaku ATX-G (Rigaku, Tokyo, Japan) operating with $\mathrm{Cu} \mathrm{K} \alpha$ radiation $(0.154 \mathrm{~nm})$. The grafted polymer density, $\sigma$ (chains $/ \mathrm{nm}^{2}$ ), of the fabricated polymer layer was calculated using the equation $\sigma=h \rho N_{A} / M_{n}$, where $h$ is the polymer layer thickness $(\mathrm{nm}), \rho$ is the polymer layer density $\left(\mathrm{g} / \mathrm{cm}^{3}\right), N_{A}$ is Avogadro constant, and $M_{n}$ is the number-average molecular weight of the free polymer chains [29].

The contact angle (CA) of the cell assay platform was measured in water to mimic an actual in vitro situation. On the cell assay platform facing downward in a transparent vessel filled with phosphate buffered saline (PBS) (Wako Pure Chemical Industries), the CA of the captured air bubble was measured by FACE CA-XP (Kyowa Interface Science, Saitama, Japan) according to the previously reported protocol [31]. Then the measured CA of the air bubble was subtracted from $180^{\circ}$ to describe the CA in this work. The PBS temperature was regulated with a temperature-controlled circulator (RE104, Lauda, Kanagawa, Japan). The result of CA was expressed as the mean of three measurements with the standard deviation (SD). The significance of CAs between $37^{\circ} \mathrm{C}$ and $20^{\circ} \mathrm{C}$ was tested by Student's $t$-test. The significance of the measured CA between samples was evaluated by the one-way analysis of variance (ANOVA).

2.4. Cells and Cell Cultures. Normal human umbilical vein ECs (Kurabo, Osaka, Japan) were maintained in HuMediaEG2 (Kurabo). Human aorta SMCs (Kurabo) were maintained in a Complete Medium Kit with Serum with CultureBoost (Cell Systems, Kirkland, WA, USA). Normal human adult dermal FBs (Kurabo) were maintained in Dulbecco's modified Eagle's medium (DMEM) (Wako Pure Chemical Industries) with $10 \%$ fetal bovine serum (Nichirei Biosciences, Tokyo, Japan) and $1 \%$ penicillin streptomycin (Wako Pure Chemical Industries). Cells were maintained at $37^{\circ} \mathrm{C}$ and $5 \% \mathrm{CO}_{2}$ and were used within five passages.

2.5. Cell Adhesion Assay. The cell adhesion assay was carried out with the previously described protocol with some modifications [29]. Viable cells stained with Calcein-AM (Dojindo Laboratories) for $1.5 \mathrm{~h}$ were seeded on the cell assay platform with droplets $\left(1.56 \times 10^{4} \mathrm{cells} / \mathrm{cm}^{2}\right)$. To switch the hydrophobic/hydrophilic property of poly(NIPAAm-coCIPAAm), the seeded platform was first cultured at $37^{\circ} \mathrm{C}$. After cell adhesion for $1 \mathrm{~h}$, cellular images were obtained on the platform by fluorescence microscopy (X71, Olympus Corporation, Tokyo, Japan) using MetaMorph (Molecular
Devices, LLC, Sunnyvale, CA, USA) controlled by an $x y z$ electric stage. Fluorescent cellular images $(N=6)$ were processed using MetaMorph to count the cellular numbers. Subsequently, the platform was transferred to a $20^{\circ} \mathrm{C}$ atmosphere for property switching from the hydrophobic status to the hydrophilic status. Upon changing the polymer status, the cells were cultured for $1 \mathrm{~h}$ for cell adhesion. Then their cellular images were obtained in the same manner. The results of cell adhesion were expressed as the mean of six cellular images with the standard deviation (SD). The significance of the data between two conditions was tested by Student's $t$-test, and the significance of the data among other conditions was tested by one-way ANOVA.

\section{Results}

3.1. Characterization of the Poly(NIPAAm-co-CIPAAm) Platform for Cell Assays. To investigate the combinational effect of a biomolecule and its immobilized polymer property, we constructed a poly(NIPAAm-co-CIPAAm)-grafted assay platform. By using thermoresponsive poly(NIPAAm-coCIPAAm), the platform can change the status of the polymer with the same immobilized biomolecules.

By measuring XRR on the assay platform, the thickness and density of the grafted layer are estimated as $3.66 \pm 0.42 \mathrm{~nm}$ and $0.95 \pm 0.02 \mathrm{~g} / \mathrm{cm}^{3}$, respectively (Table 1 ). The fabricated polymer graft has a graft density of 0.30 chains $/ \mathrm{nm}^{2}$, which reproduces the previous data $[29,32]$. The measurement of the temperature-dependent transmittance change also confirms that lower critical solution temperature (LCST) is between $20^{\circ} \mathrm{C}$ and $37^{\circ} \mathrm{C}$ (Supporting Information Figure $\mathrm{S} 1$ in Supplementary Material available online at http://dx.doi.org/10.1155/2016/2090985). This result indicates that the grafted polymer can switch its hydrophilic/hydrophobic property by thermal stimulation $\left(20^{\circ} \mathrm{C}\right.$ or $\left.37^{\circ} \mathrm{C}\right)$.

As a property descriptor, the CA was measured to describe the wettability of this platform; it measures $40.0^{\circ}$ $\left(\mathrm{SD}=0.8^{\circ}\right)$ at $20^{\circ} \mathrm{C}$ and $44.6^{\circ}\left(\mathrm{SD}=1.7^{\circ}\right)$ at $37^{\circ} \mathrm{C}$ (Figure 3$)$. Although the difference in CAs between these two temperatures is significant for all samples $\left[20^{\circ} \mathrm{C}\right.$ versus $37^{\circ} \mathrm{C}$ at blank (without biomolecules) $(p<0.05)$, mono-Arg $(p<0.05)$, mono-Ile $(p<0.01)$, tri-Arg $(p<0.001)$, and tri-Ile $(p<0.05)]$ by the $t$-test, the difference between immobilized biomolecules in the same temperature is insignificant $(p>$ 0.1 at $37^{\circ} \mathrm{C}$ and $p>0.1$ at $20^{\circ} \mathrm{C}$ ) by one-way ANOVA. Therefore, our poly(NIPAAm-co-CIPAAm) surface provides a greater property effect by thermal stimulation independent of biomolecules immobilization. 


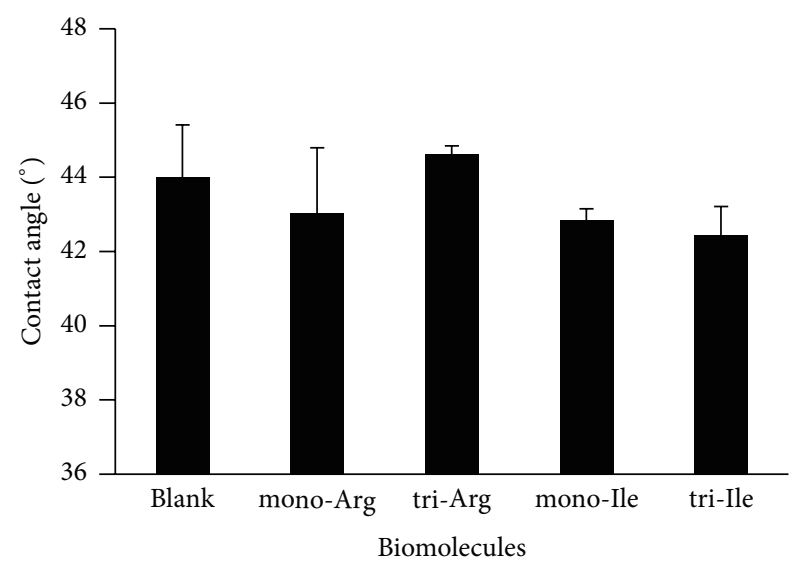

(a)

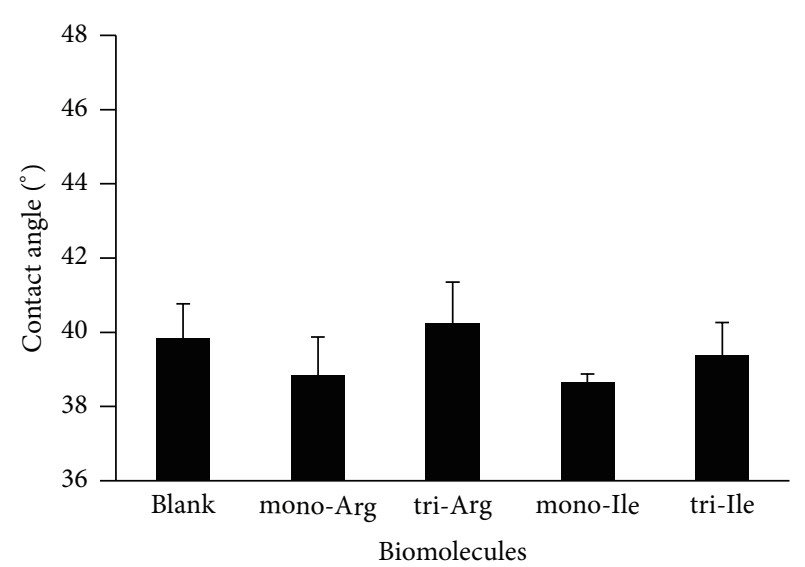

(b)

FIgUre 3: Contact angles (CAs) on the cell assay platform in this study measured at (a) $37^{\circ} \mathrm{C}$ and (b) $20^{\circ} \mathrm{C}$.

3.2. Cell Adhesion on the Biomolecule-Immobilized Poly(NIPAAm-co-CIPAAm) Platform. On the established cell assay platform, cell adhesion was assayed at either $37^{\circ} \mathrm{C}$ or $20^{\circ} \mathrm{C}$. Since the biomolecular composition and biomolecule-polymer immobilization are the same in both conditions, the impact of the different biomolecule functions on the combinational effect of the polymer property can be compared. Figure 4(a) shows the visualized cell adhesion results for the three cell types. The images $(N=6)$ are quantified by image analysis (Figure 4(b)).

First, the blank (without biomolecule) surface at $37^{\circ} \mathrm{C}$ and $20^{\circ} \mathrm{C}$ indicates that the hydrophobic property of poly(NIPAAm-co-CIPAAm) enhances cell adhesion of ECs $(p<0.05)$ and SMCs $(p<0.05)$. FBs are more insensitive to such hydrophilic/hydrophobic property changes, showing strong adhesion in both conditions. Second, the RGDSimmobilized surfaces at both $37^{\circ} \mathrm{C}$ and $20^{\circ} \mathrm{C}$ indicate that all types of cells are capable of high cell adhesion performance despite the polymer property change.

Third, mono-Arg- and tri-Arg-immobilized surfaces show similar patterns of cell adhesion for the three types of cells in both temperature conditions. Although SMCs show universally weak adhesion in all conditions, FBs show some adhesion to both mono-Arg and tri-Arg (much weaker than the RGDS peptide effect) $\left(37^{\circ} \mathrm{C}\right.$ versus $20^{\circ} \mathrm{C}: p<$ $0.05)$. ECs show weak adhesion to both mono-Arg and triArg for hydrophilic conditions at $20^{\circ} \mathrm{C}$, but its adhesion is recovered when the polymer is more hydrophobic (at $37^{\circ} \mathrm{C}$ ) $\left(37^{\circ} \mathrm{C}\right.$ versus $\left.20^{\circ} \mathrm{C}: p<0.05\right)$. A similar adhesion pattern is observed on the mono-Ile-immobilized surface with FBs. However, the adhesion performance greatly changes with ECs on the tri-Ile-immobilized surface $\left(37^{\circ} \mathrm{C}\right.$ versus $20^{\circ} \mathrm{C}$ : $p<0.01)$. When the polymer property is hydrophobic, the cell adhesion performance increases more than 2.5-fold (the ratio of EC adhesion on tri-Ile-immobilized surface at $37^{\circ} \mathrm{C}$ to $\left.20^{\circ} \mathrm{C}\right)$. Although the overall cell adhesion performance of tri-Arg and tri-Ile is much weaker than RGDS, the EC adhesion performance of tri-Ile due to the change in the polymer property is superior to RGDS.
3.3. Cell-Selective Adhesion Performance and BiomoleculePolymer Combination Effect. Compared to the universal strong cell adhesion performance of RGDS peptides, cellselective adhesion preferences between ECs, SMCs, and FBs were examined in other conditions (Figure 4(b)). The native poly(NIPAAm-co-CIPAAm) platform surface (blank) shows $\mathrm{FB}$-selective adhesion at $20^{\circ} \mathrm{C}$, but the material surface negatively affects EC and SMC adhesion (FB versus others: $p<0.01)$. Although the FB-selectivity is slightly weakened by mono-Arg immobilization at $20^{\circ} \mathrm{C}(p<0.01)$, the significantly enhanced FB-selectivity at $37^{\circ} \mathrm{C}(p<0.01)$ may be due to the combinational effect of the polymer hydrophobicity at $37^{\circ} \mathrm{C}$ (Figure $4(\mathrm{~b})$ ). In contrast, the triArg-immobilized surface also exhibits a slight FB-selectivity when the surface is hydrophilic at $20^{\circ} \mathrm{C}(p<0.01)$, which significantly weakens $\mathrm{FB}$-selectivity at $37^{\circ} \mathrm{C}$ (cell adhesion number of FBs were similar to ECs). Another enhancement of the cell-selective adhesion performance occurs with ECs on a tri-Ile-immobilized surface. The hydrophilic polymer effect enables a rather weak adhesion performance of triIle on ECs and FBs. However, when the hydrophobicity of the polymer increases, the adhesion performance to ECs is enhanced greatly, resulting in a 14.6-fold increase in the selective performance $(p<0.01)$ to discriminate between ECs and SMCs (the ratio of EC adhesion to SMC adhesion on tri-Ile-immobilized surface at $37^{\circ} \mathrm{C}$ ). These results indicate that there are effective combinational effects between an immobilized biomolecule and its immobilized polymer.

\section{Discussion}

To provide an effective biological function on medically used polymer, biomolecular immobilization is an effective strategy. Because both the immobilized biomolecule and its immobilization polymer strongly affect cell adhesion, this study investigates whether there are combinational effects, especially on cell-selective adhesion. To compare the total cell adhesion performance of a biomolecule-immobilized 

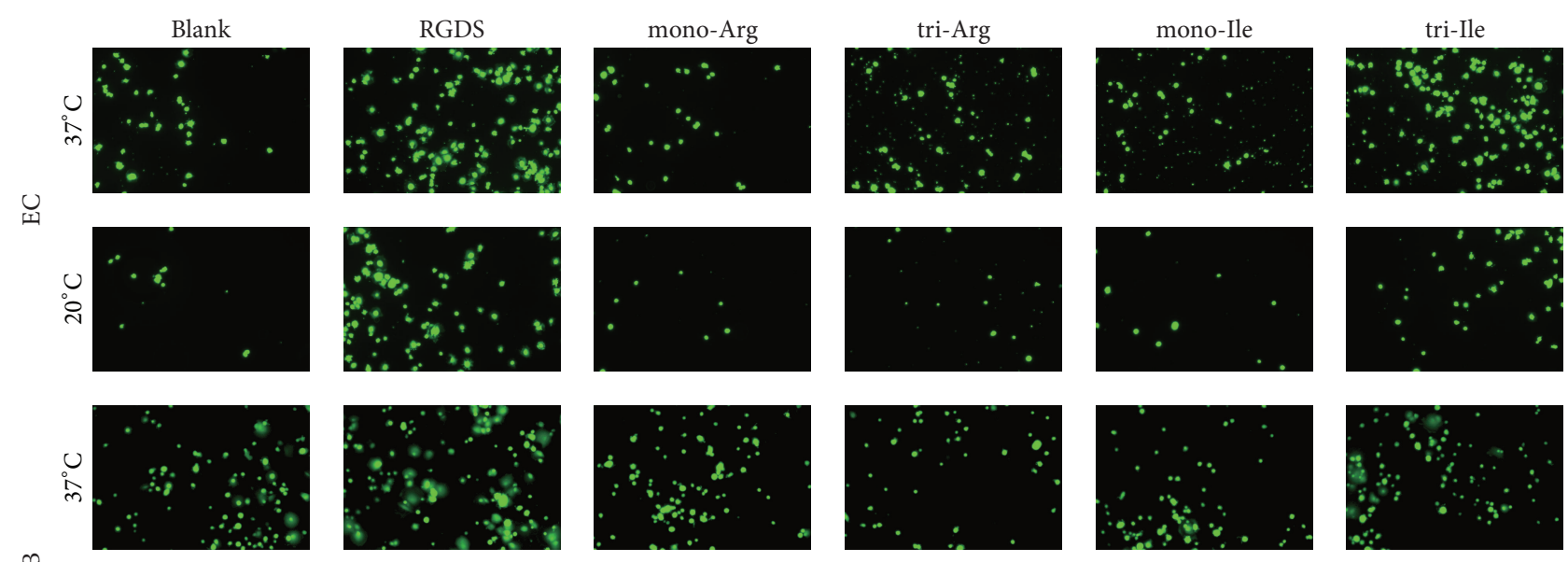

国
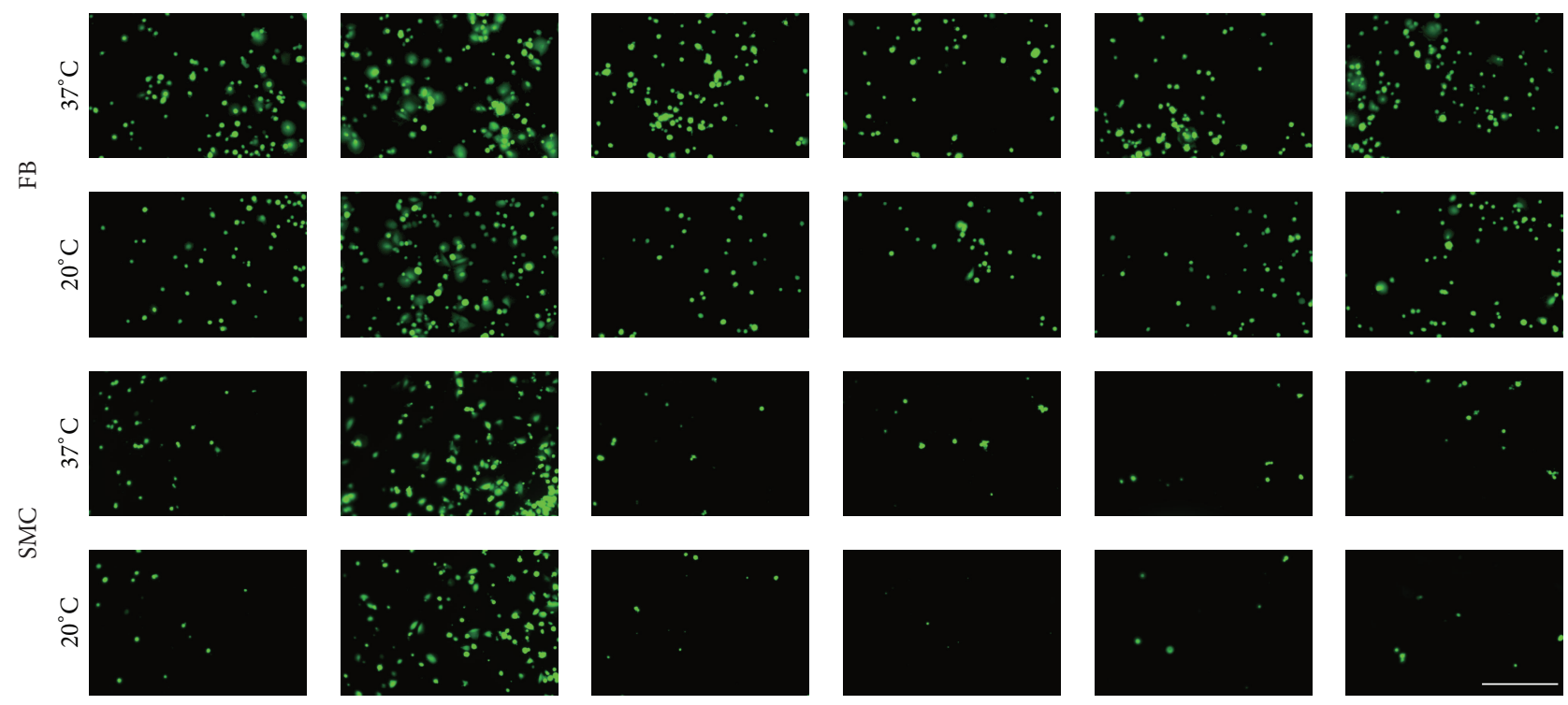

(a)

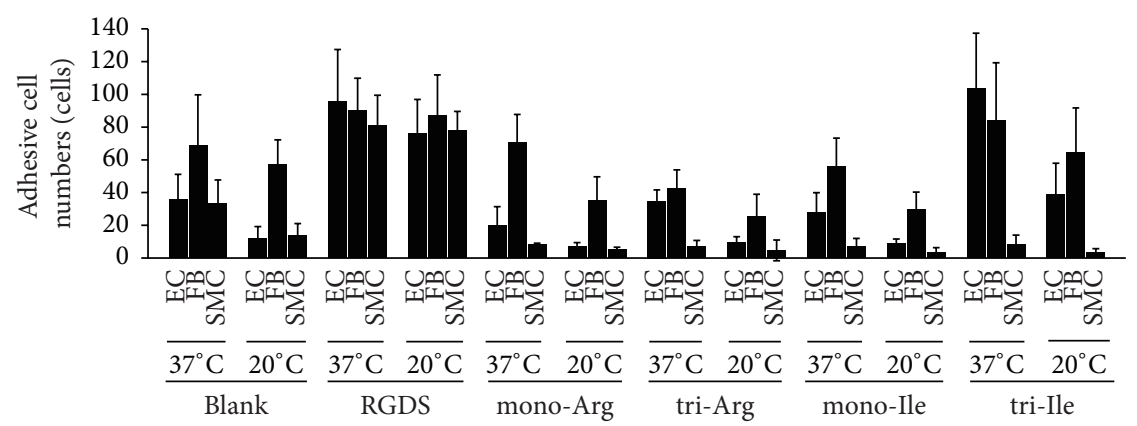

(b)

FIGURE 4: Cell adhesion performances on the biomolecule-immobilized cell assay platform. (a) Representative fluorescent image of viable cells in the cell adhesion assay (scale bar, $30 \mu \mathrm{m}$ ). (b) Quantitative results of images in (a) $(N=6)$. Blank (without biomolecules) and RGDS (positive control).

polymer by switching the property of polymer, we designed a cell assay platform using a thermoresponsive polymer.

CA, which is an indicator of the wettability of a biomaterial, has been studied; there are some wettability areas that are preferred for cell adhesion [23]. Additionally, for the plastic culture plates on the market, their surface wettability is controlled to achieve certain condition. For example, when we examined several commercially available tissue culturetreated multiwell plates (6-well plates) as basic data, CA in the air state ranges from $49.3^{\circ}$ to $93.8^{\circ}$ (plate 1: Greiner bio-one,
Cellcoat Collagen Type I, cat. 657950, lot 13-29-02-89) 47.0 $\left(\mathrm{SD}=1.0^{\circ}\right)$, (plate 2: BD Biosciences, BD PureCoat Amine 6-well plate, cat. 4721 , lot 3053352$) 49.3^{\circ}\left(\mathrm{SD}=2.4^{\circ}\right.$ ), (plate 3: Corning, Corning CellBIND Surface Polystyrene Sterile, cat. 3335 , lot 22814004) $53^{\circ}\left(\mathrm{SD}=2.5^{\circ}\right)$, (plate 4: Greiner bioone, 6 Well Advanced TC Plate, cat. 657960, lot E14113SB) $70.5^{\circ}\left(\mathrm{SD}=2.6^{\circ}\right)$, (plate 5: Falcon, Multiwell PRIMARIA 6well, cat. 353846 , lot 3269538) 83.9 $\left(\mathrm{SD}=1.7^{\circ}\right.$ ), (plate 6: Falcon, Multiwell 6-well, cat. 353046, lot 1272703) $85.9^{\circ}(\mathrm{SD}=$ $\left.5.6^{\circ}\right)$, (plate 7: Greiner bio-one, 6 Well Cell Culture Plate 
sterile with lid, cat. 657160 , lot E9070HI) $88.8^{\circ}\left(\mathrm{SD}=2.2^{\circ}\right)$, and (plate 8: Thermo Fisher Scientific, Nunclon Delta Surface, cat. 140675 , lot 7117287$) 93.8^{\circ}\left(\mathrm{SD}=0.9^{\circ}\right)$. Because all of the products show a good cell culture performance, we could not find a simple correlation between CA and cell adhesion performance. The reality is that the data to explain such rules between cell adhesion performance and the polymer surface property is limited. In other words, since there are several varieties of commercially available polymers that can culture cells with different CAs, it is extremely difficult to select the appropriate culture plate to evaluate the expected performance of immobilized biomolecules on its surface. Vast combinations of plates and immobilization conditions had to be assessed to evaluate the function of immobilized screened biomolecules.

In this work, our data provides the possibility that an immobilized biomolecule, such as an amino acid or peptide, is greatly affected by the combinational effect of the immobilized polymer property. Although our platform shows small CA differences between its hydrophilic/hydrophobic statuses, such a combinational effect is large, especially in enhancing cell-selective adhesion.

Proteins included in the serum that adsorb on the polymer surface are always an influential factor in cell adhesion. We evaluated the adsorption rates of two types of ECMderived proteins, fibronectin and collagen type IV, on our cell assay platform (Supporting Information Table S1). Our data indicates that the amount of protein adsorption is insignificant on our platform. Additionally, our previous research indicated that cell-selective adhesion peptides maintain their effects in a cell adhesion test with a serum containing medium [19]. Therefore, we consider that our cell adhesion assay data predominantly reflects the biomolecule-polymer combination effect.

Through this study, we show that the cell adhesion function of biomolecules can be greatly influenced by the combination of the biomolecule-immobilized polymer property. Interestingly, the cell-selective adhesion performance is more strongly influenced by the immobilized polymer effect. We have shown that such cell-selective adhesion can be provided not only by the peptide sequence, but also by the physicochemical property of the peptides [19]. In particular, our data shows that Ile-containing peptides can exhibit ECselective adhesion and inhibit adhesion of SMCs and FBs [19]. Accordingly, this study employs physicochemical characteristic molecules, such as Arg-containing molecule (positively charged) and Ile-containing molecule (hydrophobic). The positive charge provided by Arg is preferred by FBs; however, the effect of tri-Arg is smaller than mono-Arg at both polymer conditions $\left(37^{\circ} \mathrm{C}\right.$ and $\left.20^{\circ} \mathrm{C}\right)$. The hydrophobicity provided by Ile is preferred by both ECs and FBs, but the selectivity is greatly enhanced by the combinational effect with the polymer hydrophobicity. It is noteworthy that the EC-selectivity of tri-Ile reproduces our past results [19]. Although the difference between mono-Ile and tri-Ile at the same temperature is insignificant in the macroscopic characterization (e.g., CA), ECs can recognize the difference in the number of Ile residues and preferably adhere to tri-Ile rather than mono-Ile ( $p<0.05$ at both temperatures).
Since we have been screening cell-selective adhesion peptides with peptide microarrays [18-21], our next challenge is to select a better polymer to maximize each peptide function for medical usages. We also plan to apply our tri-Ileimmobilized surface to label-free cell purification. By using the EC-selective capture performance at $37^{\circ} \mathrm{C}$ with its EC release performance at $20^{\circ} \mathrm{C}$ in only $2 \mathrm{~h}$, it can serve as a feasible EC condensation protocol in primary cultures.

In this work, we focus on the surface wettability to characterize our platform surface because a hydrophobicity/hydrophilicity switch is the most dominant change in poly(NIPAAm-co-CIPAAm). However, other descriptors can describe the biomolecule-polymer combinational effect, such as the surface charge, rigidity, or roughness. We actually measured the zeta potentials of biomolecule-immobilized surfaces by DelsaNano HC Particle Analyzer (Beckman Coulter Inc., Brea, CA, USA). However, we did not find a clear correlation with its charged rates compared to our present CA data (Supporting Information Figure S2). Since our data suggests that the design of biomolecule-immobilized polymer materials can be more integrated via a detailed examination of combinational effects, our next objective is to extend this combinational effect investigation by evaluating the influence of other polymer properties characteristics.

\section{Conclusion}

To design an improved cell-selective adhesion function on medically used biomaterials, the combinational effect of surface-immobilized small biomolecules and the immobilization polymer property were studied. By establishing the poly(NIPAAm-co-CIPAAm) platform that changes the polymer property with the same biomolecule-polymer composition for cell adhesion assay, we found that the cellselective adhesion performance is greatly affected by the hydrophobicity of the polymer. These results suggest that appropriately selecting the polymer is extremely important to functionalize immobilized biomolecules. Hence, this work should be important for designing functionalized biomaterials for advanced regenerative therapies.

\section{Competing Interests}

The authors declare that they have no competing interests.

\section{Acknowledgments}

Part of this work was supported by Nanotechnology Platform Program (Molecule and Material Synthesis) of the Ministry of Education, Culture, Sports, Science and Technology (MEXT), Japan. Rio Kurimoto acknowledges technical support of T. Takeshima and R. Oguri (Nagoya University).

\section{References}

[1] S. Lu, X. Sun, P. Zhang, L. Yang, F. Gong, and C. Wang, "Local hemodynamic disturbance accelerates early thrombosis of small-caliber expanded polytetrafluoroethylene grafts," Perfusion (United Kingdom), vol. 28, no. 5, pp. 440-448, 2013. 
[2] D. Pfeiffer, C. Stefanitsch, K. Wankhammer et al., "Endothelialization of electrospun polycaprolactone (PCL) small caliber vascular grafts spun from different polymer blends," Journal of Biomedical Materials Research-Part A, vol. 102, no. 12, pp. 4500-4509, 2014.

[3] X. Bu, Y. Yan, Z. Zhang et al., "Properties of extracellular matrixlike scaffolds for the growth and differentiation of endothelial progenitor cells," Journal of Surgical Research, vol. 164, no. 1, pp. 50-57, 2010.

[4] K. O. Cardinal and S. K. Williams, "Assessment of the intimal response to a protein-modified stent in a tissue-engineered blood vessel mimic," Tissue Engineering_Part A, vol. 15, no. 12, pp. 3869-3876, 2009.

[5] M. Rafat, T. Matsuura, F. Li, and M. Griffith, "Surface modification of collagen-based artificial cornea for reduced endothelialization," Journal of Biomedical Materials Research Part A, vol. 88, no. 3, pp. 755-768, 2009.

[6] Y. Yu, Y. Gao, H. Wang et al., "The matrix protein CCN1 (CYR61) promotes proliferation, migration and tube formation of endothelial progenitor cells," Experimental Cell Research, vol. 314, no. 17, pp. 3198-3208, 2008.

[7] J. Aoki, P. W. Serruys, H. van Beusekom et al., "Endothelial progenitor cell capture by stents coated with antibody against CD34: the HEALING-FIM (healthy endothelial accelerated lining inhibits neointimal growth-first in man) registry," Journal of the American College of Cardiology, vol. 45, no. 10, pp. 15741579, 2005.

[8] M. D. Pierschbacher and E. Ruoslahti, "Cell attachment activity of fibronectin can be duplicated by small synthetic fragments of the molecule," Nature, vol. 309, no. 5963, pp. 30-33, 1984.

[9] R. Blindt, F. Vogt, I. Astafieva et al., "A novel drug-eluting stent coated with an integrin-binding cyclic Arg-Gly-Asp peptide inhibits neointimal hyperplasia by recruiting endothelial progenitor cells," Journal of the American College of Cardiology, vol. 47, no. 9, pp. 1786-1795, 2006.

[10] J. Graf, R. C. Ogle, F. A. Robey et al., "A pentapeptide from the laminin B1 chain mediates cell-adhesion and binds the 67000 laminin receptor," Biochemistry, vol. 26, no. 22, pp. 6896-6900, 1987.

[11] A. Komoriya, L. J. Green, M. Mervic, S. S. Yamada, K. M. Yamada, and M. J. Humphries, "The minimal essential sequence for a major cell type-specific adhesion site (CS1) within the alternatively spliced type-III connecting segment domain of fibronectin is leucine-aspartic acid-valine," The Journal of Biological Chemistry, vol. 266, no. 23, pp. 15075-15079, 1991.

[12] C. C. Larsen, F. Kligman, C. Tang, K. Kottke-Marchant, and R. E. Marchant, "A biomimetic peptide fluorosurfactant polymer for endothelialization of ePTFE with limited platelet adhesion," Biomaterials, vol. 28, no. 24, pp. 3537-3548, 2007.

[13] M. Yin, Y. Yuan, C. Liu, and J. Wang, "Development of mussel adhesive polypeptide mimics coating for in-situ inducing reendothelialization of intravascular stent devices," Biomaterials, vol. 30, no. 14, pp. 2764-2773, 2009.

[14] N. Werner, S. Junk, U. Laufs et al., "Intravenous transfusion of endothelial progenitor cells reduces neointima formation after vascular injury," Circulation Research, vol. 93, no. 2, pp. e17-e24, 2003.

[15] D. Kong, L. G. Melo, A. A. Mangi et al., "Enhanced inhibition of neointimal hyperplasia by genetically engineered endothelial progenitor cells," Circulation, vol. 109, no. 14, pp. 1769-1775, 2004.
[16] A. K. Lau, S. B. Leichtweis, P. Hume et al., "Probucol promotes functional reendothelialization in balloon-injured rabbit aortas," Circulation, vol. 107, no. 15, pp. 2031-2036, 2003.

[17] B. Li, J. X. Chen, and J. H.-C. Wang, "RGD peptide-conjugated poly(dimethylsiloxane) promotes adhesion, proliferation, and collagen secretion of human fibroblasts," Journal of Biomedical Materials Research Part A, vol. 79, no. 4, pp. 989-998, 2006.

[18] R. Kato, C. Kaga, K. Kanie, M. Kunimatsu, M. Okochi, and H. Honda, "Peptide array-based peptide-cell interaction analysis," Mini-Reviews in Organic Chemistry, vol. 8, no. 2, pp. 171-177, 2011.

[19] K. Kanie, R. Kato, Y. Z. Zhao, Y. Narita, M. Okochi, and H. Honda, "Amino acid sequence preferences to control cellspecific organization of endothelial cells, smooth muscle cells, and fibroblasts," Journal of Peptide Science, vol. 17, no. 6, pp. 479486, 2011.

[20] K. Kanie, Y. Narita, Y. Zhao et al., "Collagen type IV-specific tripeptides for selective adhesion of endothelial and smooth muscle cells," Biotechnology and Bioengineering, vol. 109, no. 7, pp. 1808-1816, 2012.

[21] F. Kuwabara, Y. Narita, A. Yamawaki-Ogata et al., "Novel smallcaliber vascular grafts with trimeric peptide for acceleration of endothelialization," Annals of Thoracic Surgery, vol. 93, no. 1, pp. 156-163, 2012.

[22] M. Khan, J. Yang, C. Shi, J. Lv, Y. Feng, and W. Zhang, "Surface tailoring for selective endothelialization and platelet inhibition via a combination of SI-ATRP and click chemistry using CysAla-Gly-peptide," Acta Biomaterialia, vol. 20, pp. 69-81, 2015.

[23] Y. Tamada and Y. Ikada, "Cell attachment to various polymer surfaces," in Polymers in Medicine II, E. Chiellini, P. Giusti, C. Migliaresi, and L. Nicolais, Eds., vol. 34, pp. 101-115, Springer US, 1986.

[24] T. Yeung, P. C. Georges, L. A. Flanagan et al., "Effects of substrate stiffness on cell morphology, cytoskeletal structure, and adhesion," Cell Motility and the Cytoskeleton, vol. 60, no. 1, pp. 24-34, 2005.

[25] D. Lehnert, B. Wehrle-Haller, C. David et al., "Cell behaviour on micropatterned substrata: limits of extracellular matrix geometry for spreading and adhesion," Journal of Cell Science, vol. 117, no. 1, pp. 41-52, 2004.

[26] M. Ebara, M. Yamato, T. Aoyagi, A. Kikuchi, K. Sakai, and T. Okano, "A novel approach to observing synergy effects of PHSRN on integrin-RGD binding using intelligent surfaces," Advanced Materials, vol. 20, no. 16, pp. 3034-3038, 2008.

[27] M. Ebara, M. Yamato, T. Aoyagi, A. Kikuchi, K. Sakai, and T. Okano, "Temperature-responsive cell culture surfaces enable 'on-off' affinity control between cell integrins and RGDS ligands," Biomacromolecules, vol. 5, no. 2, pp. 505-510, 2004.

[28] M. Ebara, M. Yamato, T. Aoyagi, A. Kikuchi, K. Sakai, and T. Okano, "The effect of extensible PEG tethers on shielding between grafted thermo-responsive polymer chains and integrin-RGD binding," Biomaterials, vol. 29, no. 27, pp. 3650$3655,2008$.

[29] N. Idota, M. Ebara, Y. Kotsuchibashi, R. Narain, and T. Aoyagi, "Novel temperature-responsive polymer brushes with carbohydrate residues facilitate selective adhesion and collection of hepatocytes," Science and Technology of Advanced Materials, vol. 13, no. 6, Article ID 064206, 2012.

[30] T. Aoyagi, M. Ebara, K. Sakai, Y. Sakurai, and T. Okano, "Novel bifunctional polymer with reactivity and temperature sensitivity," Journal of Biomaterials Science-Polymer Edition, vol. 11, no. 1, pp. 101-110, 2000. 
[31] H. Suzuki, H. M. Nurul, T. Seki et al., "Precise synthesis and physicochemical properties of high-density polymer brushes designed with poly(N-isopropylacrylamide)," Macromolecules, vol. 43, no. 23, pp. 9945-9956, 2010.

[32] K. Nagase, A. Kimura, T. Shimizu et al., "Dynamically cell separating thermo-functional biointerfaces with densely packed polymer brushes," Journal of Materials Chemistry, vol. 22, no. 37, pp. 19514-19522, 2012. 

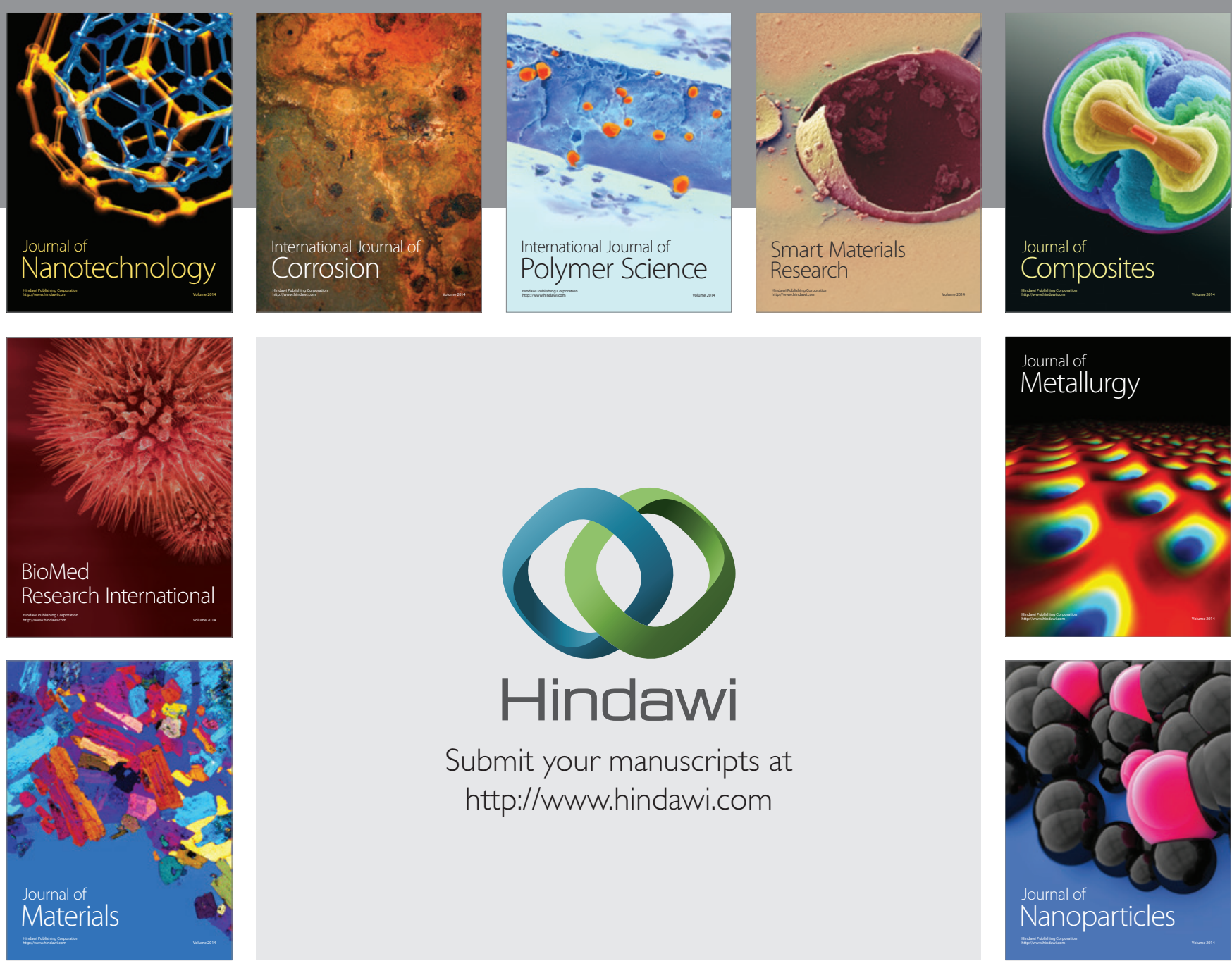

\section{Hindawi}

Submit your manuscripts at

http://www.hindawi.com

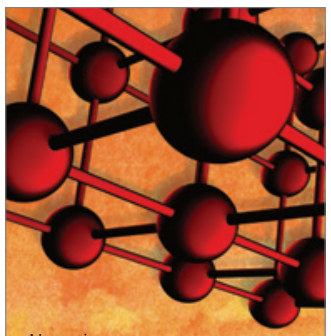

Materials Science and Engineering
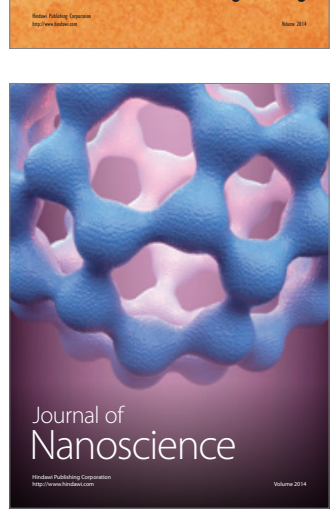
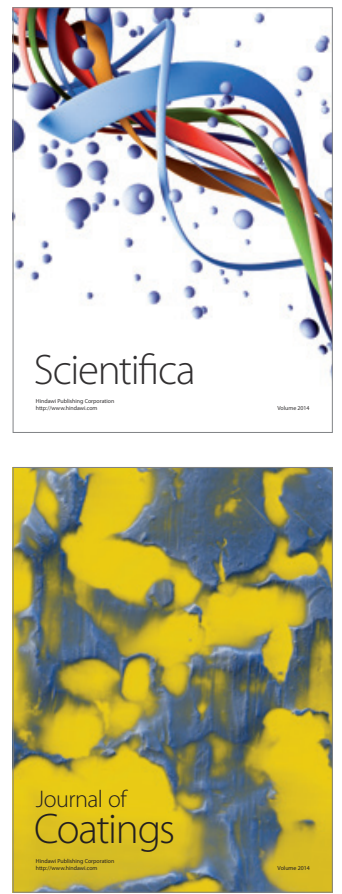
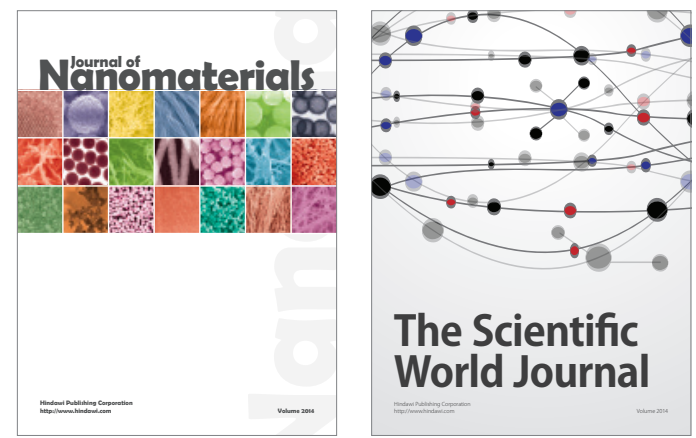

The Scientific World Journal
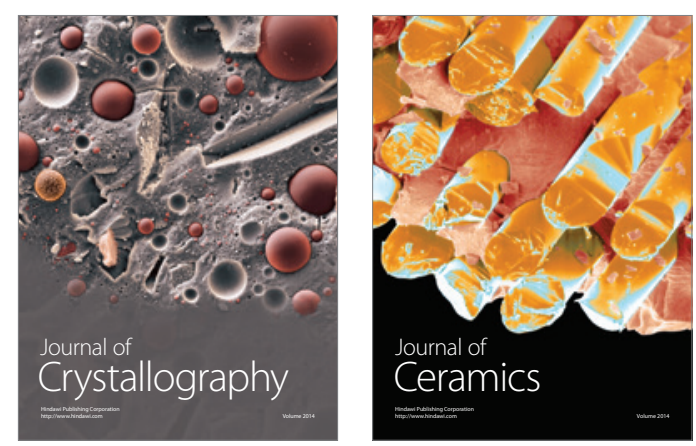
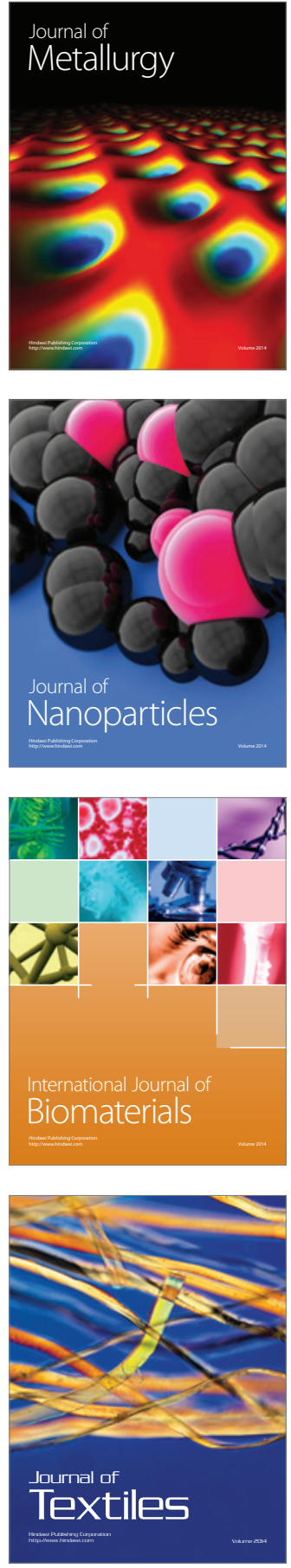